\title{
Arsenic induces Th1/Th2 imbalance in immune and non-immune organs
}

\author{
Y.Y. Guo ${ }^{1}$, L. Zhao ${ }^{1}$, S. Yang ${ }^{1}$, G.F. Sun ${ }^{1}$, B. Li $^{1}$, X.X. Duan ${ }^{2} \&$ J.L. Li ${ }^{3}$ \\ ${ }^{1}$ Environment and Non-Communicable Disease Research Center, Key Laboratory of Arsenic-related Biological \\ Effects and Prevention and Treatment in Liaoning Province, School of Public Health, China Medical University, \\ Shenyang, P.R. China \\ ${ }^{2}$ Department of Toxicology, School of Public Health, Shenyang Medical College, Shenyang, Liaoning, P.R. China \\ ${ }^{3}$ Department of Occupational and Environmental Health, Key Laboratory of Occupational Health and Safety for \\ Coal Industry in Hebei Province, School of Public Health, North China University of Science and Technology, \\ Tangshan, Hebei, P.R. China
}

\begin{abstract}
CD4+ T helper (Th) cells, especially well balanced Th1/Th2 responses, are essential regulators of the immune responses and inflammatory diseases. Arsenic is a known immunosuppressive metalloid, but its detailed effects on immune and non-immune organs are poorly understood. In the present study, mice were treated with $2.5,5$ and $10 \mathrm{mg} \mathrm{kg}^{-1} \mathrm{NaAsO}_{2}$ intra-gastrically for $24 \mathrm{~h}$. We found that arsenic exposure led to the persistent aberration of the inherent capability of Th cells to differentiate into Th1 and Th2 cells. These results might provide novel therapeutic strategies on arsenic-induced immune related diseases.
\end{abstract}

\section{INTRODUCTION}

Arsenic is the number one contaminant of concern for human health worldwide according to the Agency for Toxic Substances and Disease Registry (ATSDR, 2007). Micromolar concentrations of arsenic could not only reduce the differentiation of human peripheral blood mononuclear cells (PBMCs) into functional macrophages (Lemarie et al., 2006a), but also reverse the major phenotypic and genetic features of human mature macrophages (Lemarie et al., 2006b). What's more, the balance of the earliest determined Th cell subsets, Th1 and Th2, play important roles in immune function. In the present study, we set up arsenic exposure models by treating mice with an oral administration of $2.5,5$ and $10 \mathrm{mg} \mathrm{kg}^{-1} \mathrm{NaAsO}_{2}$, and determined the Th1/Th2 differentiation in spleen, thymus and lung. The present study was undertaken to comprehensively assess the immunomodulatory effect of arsenic exposure in vivo.

\section{METHODS/EXPERIMENTAL}

\subsection{Animals and experimental procedures}

Forty female C57BL/6 mice (weighing 18-22 g, 67 weeks old) were obtained from the Center for Experimental Animals at China Medical University (Shenyang, China). Mice were exposed to environmentally relevant concentrations of $\mathrm{NaAsO}_{2}(2.5,5$ and $10 \mathrm{mg} \mathrm{kg}^{-1}$ ) intragastrically for $24 \mathrm{~h}$, respectively. At autopsy, the entire spleen, thymus and lung were promptly removed, weighed, and stored at $-80^{\circ} \mathrm{C}$ for future use.

\subsection{Determination of tissue arsenic levels}

Spleen, thymus and lung were homogenized on ice with $500 \mu \mathrm{L}$ deionized water and $0.05 \mathrm{~g}$ tissues. Determination of arsenic species, including iAs, monomethylarsonic acid (MMA) and dimethylarsinic acid (DMA), were determined by HPLC-HG-AFS (SA-10 Atomic Fluorescence Species Analyzer, Titan Co, Beijing). Total arsenic (T-As) levels were then calculated by summing up the levels of iAs, MMA and DMA. All samples were analyzed in triplicate, and the results were expressed as mean $\pm \operatorname{SD}(n=4)$.

\subsection{Total RNA isolation and qPCR analysis}

Total RNA of spleen, thymus and lung from experimental mice was isolated using a Trizol Reagent (Invitrogen). Real-time PCR was conducted using a two-step method with an ABI 7500 Real-Time PCR System (ABI, USA).

\subsection{Statistical analysis}

Data were presented as mean \pm standard deviation (SD). Statistical significance was determined by ANOVA. P values of less than 0.05 were considered as statistically significant. 
Table 1. Total arsenic (T-As, $\mu \mathrm{g} \mathrm{As} \mathrm{g}^{-1}$ tissue) levels and weights in spleen, thymus and lung of control and different experimental mice.

\begin{tabular}{lllllll}
\hline $\mathrm{NaAsO}_{2}\left(\mathrm{mg} \mathrm{kg}^{-1}\right)$ & T-As in spleen & T-As in thymus & T-As in lung & Spleen weight & Thymus weight & Lung weight \\
\hline control & $<\mathrm{LD}$ & $<\mathrm{LD}$ & $<\mathrm{LD}$ & $0.07 \pm 0.01$ & $0.07 \pm 0.01$ & $0.13 \pm 0.01$ \\
2.5 & $0.95 \pm 0.08$ & $1.21 \pm 0.46$ & $0.35 \pm 0.02$ & $0.06 \pm 0.01^{*}$ & $0.05 \pm 0.01^{*}$ & $0.14 \pm 0.01$ \\
5 & $1.51 \pm 0.06$ & $1.82 \pm 0.72$ & $0.91 \pm 0.17$ & $0.05 \pm 0.01^{*}$ & $0.04 \pm 0.01^{*}$ & $0.13 \pm 0.01$ \\
10 & $2.73 \pm 0.31$ & $3.01 \pm 0.56$ & $2.2 \pm 0.29$ & $0.05 \pm 0.01^{*}$ & $0.04 \pm 0.01^{*}$ & $0.13 \pm 0.01$ \\
\hline
\end{tabular}

(A)

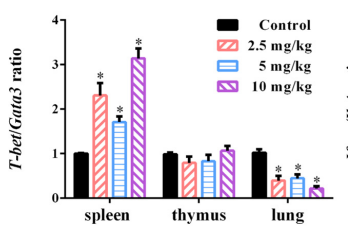

(B)

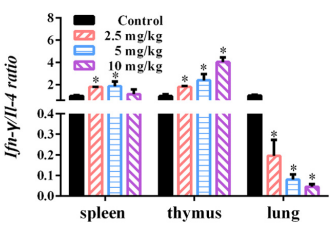

Figure 1. Effects of arsenic exposure on Th1/Th2 differentiation in spleen, thymus and lung of control and different experimental mice. T-bet/Gata3 ratio and Ifn- $\gamma / \mathrm{Il}-4$ ratio were determined and calculated, respectively. $*$ denoted $\mathrm{p}<0.05$ compared with control.

\section{RESULTS AND DISCUSSION}

\subsection{T-As levels and weights of spleen, thymus and lung by arsenic exposure}

In our study, no significant difference of body weight, as well as general status were observed among all the study mice, suggesting no obvious growth inhibition of the experimental mice (data not shown). We detected T-As levels at $12 \mathrm{~h}$ and found that T-As levels were significantly dose-dependent in different arsenic-treatment group (Table 1). We only found that the splenic and thymic weights in all arsenic-treated groups were consistently lower than in control group, suggesting arsenic induced immunotoxicity in mice.

\subsection{Arsenic exposure regulates Th1/Th2 differentiation in spleen, thymus and lung}

Recent years, the Th1/Th2 immune balance is thought to exert critical roles in stabilize microenvironment in vivo (Moriyama and Nakamura, 2005). Our results here observed an increase in T-bet/Gata3 ratio of spleen, and a decrease in lung. Ifn- $\gamma / \mathrm{Il}-4$ ratio were also moderately upregulated both in spleen and thymus, as well as significantly decreased in lung (Fig. 1). Our results indicate that arsenic could induce an imbalance of Th1/Th2 differentiation and Th1polarized immune responses in spleen and thymus, while Th2-polarized immune responses in lung.

\section{CONCLUSIONS}

In conclusion, our present study demonstrated that arsenic-induced immune changes are associated with modulation of Th1/Th2 differentiation in spleen, thymus and lung of $\mathrm{C} 57 \mathrm{BL} / 6$ mice.

\section{ACKNOWLEDGEMENTS}

This study was supported by grants from National Natural Science Foundation China (NSFC, No. 81673114) and Key Laboratory Basic Research Funds from Liaoning Education Department (LS201607).

\section{REFERENCES}

ATSDR, 2007. Toxicological Profile for Arsenic (Update). Agency for Toxic Substances and Disease Registry. Atlanta, GA.

Lemarie, A., Morzadec, C., Merino, D., Bourdonnay, E., Fardel, O. \& Vernhet, L. 2006a. Human macrophages constitute targets for immunotoxic inorganic arsenic. $J$. Immunol. 177 (5): 3019-3027.

Lemarie, A., Morzadec, C., Merino, D., Micheau, O., Fardel, O. \& Vernhet, L. 2006b. Arsenic trioxide induces apoptosis of human monocytes during macrophagic differentiation through nuclear factor-KappaB-related survival pathway down-regulation. J. Pharmacol. Exp. Ther. 316(1): 304314.

Moriyama, M. \& Nakamura, S. 2016. Th1/Th2 immune balance and other T helper subsets in IgG4-related disease. Curr Top. Microbiol. Immunol. 401: 75-83. 